\title{
Detección de problemas de salud mental en un grupo especialmente vulnerable: niños y adolescentes en acogimiento residencial
}

\author{
Ana Sainero, Jorge F. del Valle* y Amaia Bravo
}

Universidad de Oviedo, Grupo de Investigación en Familia e Infancia

\begin{abstract}
Resumen: La investigación sobre la salud mental de niños y adolescentes en acogimiento residencial muestra una alta incidencia de trastornos, particularmente de tipo externalizante, y por consiguiente es muy importante utilizar instrumentos de detección que permitan intervenciones tempranas. El objetivo de este estudio fue describir las características y analizar el grado de concordancia en la información que ofrecen los jóvenes y sus educadores empleando la técnica de screening del CBCL (para los educadores) y el YSR (para los adolescentes) sobre una muestra de 138 adolescentes entre 11 y 18 años en hogares de protección. Se analizaron también las diferencias entre el grupo de jóvenes derivados a tratamiento de salud mental y aquellos que no lo recibían. Los resultados mostraron niveles de acuerdo bajos y moderados entre jóvenes y educadores, con mayor concordancia para problemas externalizantes que internalizantes, de forma similar a lo hallado con muestras de padres e hijos. Las diferencias de puntuaciones se discuten en relación a la investigación revisada y se concluye que los adultos distinguen al grupo clínico del que no lo es, mientras los jóvenes no presentan diferencias significativas. Estos hallazgos indicarían la necesidad de dar más relevancia al autoinforme, a la perspectiva de los adolescentes.

Palabras clave: Salud mental; acogimiento residencial; CBCL, YSR.
\end{abstract}

\section{Introducción}

La salud mental infanto-juvenil es uno de los temas prioritarios en las estrategias de salud y uno de los temas pendientes de mejora y desarrollo en España (Castro-Fornieles, 2013). Dentro de esta población infanto-juvenil existe un grupo especialmente vulnerable que no siempre ha recibido la suficiente atención (Muela, Balluerka y Torres, 2013). Se trata de aquellos menores de edad que se encuentran en situación de riesgo o desamparo (tal como lo define nuestro Código Civil) por haber sufrido malos tratos o abandono en su contexto familiar. En España tenemos más 30.000 niños y adolescentes que han sido separados de sus familias y acogidos en familias o establecimientos residenciales (Observatorio de la Infancia, 2012) para su protección y seguridad (véase una revisión de las características de estas intervenciones en España en Bravo y Del Valle (2009) y Del Valle, Bravo y López (2009).

La incidencia de problemas de salud mental en esta población es muy elevada como cabe imaginar, y así se ha constatado ampliamente en la literatura científica (Burns et al., 2004; Clausen, Landsverk, Ganger, Chadwick, y Litrownik, 1998; Farmer et al., 2001; Garland, Landsverk, y Lau, 2003; Heflinger, Simpkins, y Combs-Orme, 2000; Landsverk, Burns, Stambaugh, y Rolls-Reutz, 2006; Pecora, Jensen, Romanelli, Jackson, y Ortiz, 2009; Tarren-Sweeney y Hazell, 2006). En el caso de Europa, un reciente estudio

* Dirección para correspondencia [Correspondence address]:

Jorge F. del Valle. Facultad de Psicología, Universidad de Oviedo, Plaza Feijoo s/n, 33003 Oviedo (España).E-mail: jvalle@uniovi.es
Title: Detection of mental health problems in an especially vulnerable group: children and young people in residential child care.

Abstract: Research on mental health problems of children and young people in residential child care shows a high incidence. One of the strategies to improve the interventions is the use of tools of detection, so that biases in the referral to treatment could be avoided.

The objective of this study was to analyze the level of concordance between the information given by the young people and their social educators, using the CBCL (in case of educators) and YSR (for adolescents) in a sample of 138 young people aged from 11 to 18 who were in residential care. Also differences between the group of young people referred to mental health treatment and those without it were analyzed.

Results show low and moderate levels of concordance between the information given by the young people and their educators, with higher levels of agreement in externalized problems, in a similar way as the results found in research with samples of parents and children. Score differences are discussed according to the literature review, concluding that adults distinguish the clinical and non-clinical group clearly, but young people do not display significant differences. These findings indicate the need for giving more relevance to self-reports, to the adolescent own perspective.

Key words: mental health; residential child care; CBCL, YSR.

francés indica que el $48 \%$ de los niños en acogimiento residencial presentan algún tipo de trastorno (Bronsard et al., 2011). En Gran Bretaña se han presentado datos en este mismo sentido, afirmando que existe una proporción de casos con trastornos cuatro veces mayor que en la población general (Ford, Vostanis, Meltzer, y Goodman, 2007; Nicholas, Roberts, y Wurr, 2003; Sempik, Ward, y Darker, 2008). A similares conclusiones se ha llegado por estudios llevados a cabo en otros países europeos (European Association for Research into Residential Child Care, 1998; Holtan, Ronning, Handegard, y Sourander, 2005; Hukkanen, Sourander, Santalahti, y Bergroth, 2005). Finalmente, en el único estudio realizado en España, se muestra que el $27 \%$ de niños y jóvenes entre 6 y 18 años acogidos en residencias está recibiendo tratamiento debido a problemas de salud mental (Sainero, Bravo, y Del Valle, 2014).

La relevancia de estos datos ha llevado en Estados Unidos a la realización de un trabajo conjunto entre la American Academy of Child and Adolescent Psychiatry (AACAP) y la Child Welfare League of America (CWLA) para realizar propuestas sobre la atención de la salud mental de este grupo (Pecora et al., 2009). Se trata de un trabajo compartido entre los ámbitos del sistema de salud mental infanto-juvenil y de los servicios sociales de protección a la infancia, que es muy poco frecuente en el resto de países. Entre sus conclusiones han destacado la necesidad de una detección temprana de los problemas y un acceso fácil y fluido a servicios terapéuticos, para lo que se recomiendan instrumentos objetivos de screening que eviten que las derivaciones a tratamientos se realicen bajo criterios poco controlados (Burns, et al., 2004).

Los niños y adolescentes que se encuentran en acogi- 
miento residencial viven en hogares de protección en los que profesionales de la educación social les prestan su apoyo y se encargan de su educación integral. La detección de problemas de salud mental y la decisión de derivar hacia tratamientos depende en gran parte de estos profesionales. Sin embargo, en España tampoco se están utilizando instrumentos o medidas objetivas para ello (Sainero et al., 2013 ) a pesar de que los trastornos emocionales y de conducta de los adolescentes en acogimiento residencial está suponiendo uno de los problemas más graves de este tipo de servicios (Bravo y Del Valle, 2009; Defensor del Pueblo, 2009).

En este artículo se presenta una investigación sobre las alteraciones emocionales y conductuales de adolescentes en acogimiento residencial desde la perspectiva de la detección mediante instrumentos de screening, en concreto el modelo dimensional y multi-informante propuesto por Achenbach y Edelbrock (1983). Desde hace más de dos décadas esta propuesta se ha convertido en una de las herramientas más importantes en la detección y evaluación de los problemas emocionales y conductuales infanto-juveniles. Mediante la aplicación de instrumentos de evaluación y diagnóstico (Child Behavior Check List, Teacher Report Form y Youth Self Report) que recogen la perspectiva de los principales observadores de la conducta infantil y adolescente (padres, profesores y los propios jóvenes mayores de 11 años), se evalúa la posible existencia, tipología y gravedad de las alteraciones clínicas. Se considera que la evaluación clínica infantil debe contar con múltiples perspectivas sobre la conducta del niño y preferiblemente que informen acerca de diferentes contextos (Achenbach, 2006).

Este planteamiento de utilización de varios informantes para la evaluación de trastornos de conducta en el niño ha llevado a extensas investigaciones sobre el grado de concordancia entre ellos. En un estudio de revisión ya clásico Achenbach, McConaughy, Stephanie, y Howell (1987) hallaron correlaciones elevadas para informantes similares (por ejemplo ambos padres con una correlación media de .60) y moderadas entre diferentes informantes como padres y profesores (oscilan entre 20 y .30), siendo la existente entre padres y adolescentes de .24. Estos resultados han sido replicados confirmándose correlaciones moderadas o bajas entre las diferentes fuentes de información (Barkmann y SchultMarkwort, 2005; De los Reyes y Kazdin, 2005; Renk y Phares, 2004; Rey, Schrader, y Morris-Yates, 1992; SeiffgeKrenke y Kollmar, 1998; Stranger y Lewis, 1993; Verhulst y Van der Ende, 1992; Yeh y Weisz, 2001). A pesar de estas tendencias moderadas se considera que la información proporcionada por las diferentes partes contribuye eficazmente en la explicación de la conducta del niño (Achenbach, 2006), ya que son informaciones referidas a diferentes contextos $y$ que pueden complementarse.

Un hallazgo muy importante de la comparación entre padres e hijos como informantes es que las diferencias dependen de si se trata de adolescentes de población general o grupos clínicos. En el caso de estudios con muestras de la población general las puntuaciones de los jóvenes en el auto- informe tienden a ser más elevadas (mayor sintomatología clínica) que las puntuaciones aportadas por sus padres, especialmente en los problemas de tipo internalizante (Achenbach y Rescorla, 2001; Begovac, Rudan, Skocic, y Filipovic, 2004; Rescorla et al., 2013; Stranger y Lewis, 1993; Van der Ende y Verhulst, 2005; Verhulst y Van der Ende, 1992; Vierhaus y Lohaus, 2008). Esta tendencia, sin embargo, se invierte en estudios con muestras clínicas (casos en tratamiento), en las que las puntuaciones de los padres mediante el CBCL informan de más conductas problemáticas que las aportadas por los adolescentes en el YSR (Ferdinand, Van der Ende, y Verhulst, 2004; Kolko y Kazdin, 1993; Kristensen, 2001). Esto podría deberse a que los jóvenes con diagnóstico tienden a infravalorar la gravedad de sus síntomas mientras que sus padres tienden a ser más severos en sus informaciones (Berg-Nielsen, Vika, y Dahl, 2003).

Este artículo se centra en el uso del CBCL (cuestionario para los padres) e YSR (cuestionario para adolescentes) como instrumentos de detección de manifestaciones clínicas en acogimiento residencial. Un problema evidente es que en este escenario los adolescentes no viven con sus padres, sino con sus educadores y por tanto el CBCL debe ser completado por estos profesionales como personas responsables de su educación y que conviven con ellos diariamente. Sin embargo, apenas existen trabajos que hayan investigado la relación entre informantes cuando se trata de educadores en acogimiento residencial y adolescentes (Handwerk, Larzelere, Soper, y Friman, 1999).

El primer objetivo de este estudio es perfilar las características socio-demográficas de los adolescentes acogidos en hogares de protección. En segundo lugar se pretende analizar la concordancia entre informantes cuando se trata de la aplicación del CBCL y el YSR como herramientas de screening en acogimiento residencial (en este contexto el informante adulto no son los padres sino los educadores). Para ello se analizará, en primer lugar, el grado de concordancia entre ambas perspectivas siguiendo una línea de investigación ya clásica en estos instrumentos pero escasamente evaluada en acogimiento residencial. Posteriormente, se analizarán las diferencias halladas en las diferentes escalas clínicas de los instrumentos, en cuanto a su dirección y su magnitud y, particularmente, si se encuentran las mismas tendencias con respecto a los grupos clínicos y no clínicos (diferenciados en base a la existencia o no de derivación a servicios terapéuticos) que las halladas cuando se trata de padres e hijos.

\section{Método}

\section{Participantes}

El estudio se llevó a cabo sobre los adolescentes con edades comprendidas entre los 11 y los 18 años $(N=138)$ que vivían en acogimiento residencial en la comunidad autónoma de Extremadura y que llevaran al menos tres meses conviviendo con sus educadores (estas edades y el tiempo de 
observación son criterios necesarios para la aplicación de estas pruebas de screening).

\section{Instrumentos y procedimiento}

Se recogieron variables relativas a las características de los adolescentes y al proceso de intervención protectora que se estaba realizando. Para ello se elaboró una hoja de recogida de información que fue completada por el educador-tutor de cada adolescente, recogiendo variables descriptivas (edad, sexo) y su estado de salud, con especial énfasis en la salud mental. Se solicitaron datos sobre el historial médico (enfermedades físicas, algún tipo de discapacidad reconocida) así como información sobre la existencia de trastornos de salud mental (derivación a tratamiento, medicación, diagnóstico, etc.).

Sobre el tipo de intervención de protección se recogieron variables como el motivo de ingreso, el tiempo de permanencia o el número de cambios que había experimentado el adolescente. Esta información fue aportada por los trabajadores sociales responsables del caso en los servicios sociales de protección, completando una hoja de datos de cada caso.

Para la identificación de los problemas emocionales y conductuales se aplicaron dos instrumentos de screening de validez y fiabilidad ampliamente demostrada en la evaluación infanto-juvenil y de uso muy frecuente en niños en protección infantil: Child Behavior Check List (CBCL) y Youth Self Report (YSR) (Achenbach y Rescorla, 2001). En este caso, el CBCL fue completado por el educador-tutor para cada uno de los jóvenes, tal y como suele suceder en los estudios en acogimiento residencial. Por su parte, los adolescentes mayores de 11 años (edad mínima de aplicación) completaron autónomamente la versión de autoinforme.

Ambos instrumentos se organizan en un listado de conductas problemáticas que se valora según su grado de ocurrencia. Las puntuaciones obtenidas se agrupan en 8 escalas clínicas específicas (ansiedad/depresión, aislamiento/ depresión, quejas somáticas, problemas sociales, problemas de pensamiento, problemas de atención, conducta disruptiva y conducta agresiva) cuyas puntuaciones se combinan para formar dos escalas de segundo orden referidas a trastornos internalizantes y externalizantes, así como una escala total.

El procedimiento se llevó a cabo mediante una aplicación colectiva a los adolescentes en cada uno de los hogares de acogida por parte del equipo investigador, mientras los educadores-tutores lo completaban junto con la hoja de datos personales y de salud. El estudio contaba con los permisos pertinentes de los servicios de protección infantil de la comunidad (que ostenta la tutela de los adolescentes) y la aprobación del comité de ética de la Facultad de Psicología de la Universidad.

El análisis de datos consistió en perfilar las características de la muestra con estadísticos descriptivos y comparar los resultados obtenidos en los dos instrumentos de screening (CBCL e YSR). Esta comparación se llevó a cabo mediante dos análisis diferentes: la correlación de Pearson y la prueba $t$ de Student. Mediante la correlación de Pearson se pretende valorar el grado de acuerdo entre los informantes y la relación existente entre sus percepciones. La prueba de diferencias $t$ permite comparar las puntuaciones informadas por los educadores y adolescentes y conocer la magnitud de las variaciones que se encuentren. Al analizar diferencias separadamente para el grupo clínico y no clínico, el pequeño tamaño de la muestra del primero $(n=35)$ nos ha llevado a utilizar en su caso técnicas no paramétricas tanto para las diferencias (prueba de Wilcoxon) como para la correlación (rho de Spearman).

\section{Resultados}

La distribución de la muestra fue muy similar en cuanto al sexo (51.4\% varones) y por rangos de edad (tabla 1). De acuerdo a la clasificación de Sinclair, Baker, Lee and Gibbs (2007) que tiene en cuenta la edad y la causa de ingreso, el $39.9 \%$ había ingresado en su infancia (por debajo de los 11 años) y había alcanzado la adolescencia en acogimiento residencial, el 41.3\% habían ingresado siendo ya adolescentes con problemas de maltrato y el $18.8 \%$ ingresó como adolescente pero $\sin$ historia de maltrato.

Tabla 1. Características de la muestra

\begin{tabular}{|c|c|}
\hline Características & $\begin{array}{l}\text { Total }(N=138) \\
\% \text { or } M(D T)\end{array}$ \\
\hline \multicolumn{2}{|l|}{$\overline{\text { Sexo }}$} \\
\hline Mujer & 49 \\
\hline Varón & 51 \\
\hline \multicolumn{2}{|l|}{$\overline{\text { Edad }}$} \\
\hline$M(S D)$ & $15.3(1.5)$ \\
\hline $12-14$ & 48.6 \\
\hline $15-18$ & 51.4 \\
\hline \multicolumn{2}{|l|}{ Tipo de casos (Sinclair et al., 2007) } \\
\hline Ingreso de adolescente con maltrato & 41.3 \\
\hline Ingreso de adolescente sin maltrato & 18.8 \\
\hline Adolescente que ingresó en su infancia & 39.9 \\
\hline Tiempo de estancia (meses) & $32.6(30.5)$ \\
\hline Número de cambios de medida & $2.4(1.4)$ \\
\hline \multicolumn{2}{|l|}{ Motivo de ingreso ${ }^{\mathrm{a}}$} \\
\hline Negligencia física & 58.7 \\
\hline Negligencia emocional & 44.9 \\
\hline Maltrato físico & 17.4 \\
\hline Maltrato emocional & 21.7 \\
\hline Abuso sexual & 5.1 \\
\hline Discapacidad intelectual & 16.7 \\
\hline $\begin{array}{l}\text { Tratamiento psicológico y/o psiquiátrico (grupo } \\
\text { clínico) }\end{array}$ & 25.4 \\
\hline Tratamiento farmacológico & 13.0 \\
\hline
\end{tabular}

a: Puede haber más de un motivo

Sobre los motivos que determinaron el ingreso en protección, el más común fue la negligencia física en más de la mitad de los casos (59\%) y la emocional en el $45 \%$. La frecuencia de maltrato físico fue del $17 \%$ y de abuso sexual el $5 \%$. El tiempo medio de permanencia en el hogar de acogida fue de aproximadamente 3 años y el número de cambios o 
transiciones (casi siempre se trataba de cambios de hogar de protección, aunque en algún caso habían pasado por una familia de acogida) que habían experimentado a lo largo de su historia en protección fue de $2.4(D T=1.4)$.

En cuanto a los datos sobre salud mental, uno de cada cuatro casos $(25.4 \%)$ estaba recibiendo o había recibido recientemente (últimos dos años) tratamiento psicológico y/o psiquiátrico por problemas de salud mental. Este grupo es el que consideraremos en el resto de análisis como grupo clínico, entendiendo por tal el que había sido identificado y derivado a tratamiento por el personal de los hogares de acogida. También se halló una elevada prevalencia (16.7\%) de adolescentes con un diagnóstico de discapacidad intelectual, de los cuales un 30.4\% formaba parte del grupo clínico derivado a tratamiento. Un $13 \%$ de la muestra total estaba recibiendo tratamiento farmacológico (un 64\% de los que estaban derivados a servicios terapéuticos).

De los 35 adolescentes con tratamiento, solamente en 17 casos los educadores podían aportar un diagnóstico conocido, de ellos el 65\% pertenecían a la categoría de trastornos de tipo externalizante, frente al 12\% que tenían un diagnós- tico de tipo internalizante (en el resto de casos, se informaba de un diagnóstico muy genérico no clasificable) .

En cuanto a la comparación de resultados entre CBCL e YSR, el análisis de diferencias entre medias resultó estadísticamente significativo en todas las escalas, excepto las de conducta agresiva y conducta disruptiva (tabla 2), siempre en el sentido de una puntuación menor cuando los informantes son los educadores (CBCL). En cuanto al grado de concordancia entre las pruebas, las correlaciones entre escalas oscilaron entre $r=.10$ para problemas de pensamiento y $r=.52$ para la escala de conducta disruptiva. Todas resultaron ser estadísticamente significativas exceptuando la escalas de problemas de pensamiento, ya citada y la de problemas de atención $(r=.16)$ aunque se trata de magnitudes bajas o moderadas. Estos resultados indican que a pesar de las diferencias halladas en las respuestas de educadores y jóvenes existe una cierta relación (al menos para los problemas externalizantes) entre sus puntuaciones, de modo que a mayor puntuación de los educadores, mayor puntuación presentan los propios jóvenes.

Tabla 2. Diferencias entre medias y correlaciones en CBCL e YSR.

\begin{tabular}{|c|c|c|c|c|c|c|c|c|}
\hline & \multicolumn{2}{|c|}{ CBCL } & \multicolumn{2}{|c|}{ YSR } & \multicolumn{2}{|c|}{ Diferencia entre medias } & \multicolumn{2}{|c|}{ Correlación } \\
\hline & $M$ & $D T$ & $M$ & $D T$ & $t$ & $p$ & $r$ & $p$ \\
\hline Ansiedad/depresión & 54.03 & 6.50 & 59.36 & 8.40 & -6.67 & $.000^{* * *}$ & .23 & $.008^{* *}$ \\
\hline Aislamiento/depresión & 57.86 & 8.87 & 61.17 & 9.80 & -3.26 & $.001 * *$ & .19 & $.026^{*}$ \\
\hline Quejas somáticas & 54.09 & 6.90 & 57.98 & 8.20 & -4.69 & $.000 * * *$ & .18 & $.039 *$ \\
\hline Probl. sociales & 57.95 & 8.61 & 60.98 & 9.22 & -3.12 & $.002 * *$ & .18 & $.031 *$ \\
\hline Probl. de pensamiento & 53.36 & 6.11 & 57.62 & 8.16 & -5.15 & $.000 * * *$ & .10 & .262 \\
\hline Probl. de atención & 57.79 & 7.64 & 60.01 & 8.76 & -2.45 & $.016^{*}$ & .16 & .056 \\
\hline Conducta disruptiva & 61.04 & 8.72 & 62.38 & 9.35 & -1.78 & .077 & .52 & $.000^{* * *}$ \\
\hline Conducta agresiva & 59.38 & 10.56 & 60.42 & 9.46 & -0.98 & .327 & .24 & $.004^{* *}$ \\
\hline Escala internalizante & 51.38 & 10.10 & 59.16 & 10.30 & -6.70 & $.000^{* * *}$ & .18 & $.033^{*}$ \\
\hline Escala externalizante & 58.33 & 11.53 & 60.99 & 10.67 & -2.51 & $.013 *$ & .37 & $.000 * * *$ \\
\hline Escala total & 55.10 & 11.62 & 60.67 & 9.99 & -4.69 & $.000 * * *$ & .17 & $.042^{*}$ \\
\hline
\end{tabular}

$*: p<.05 ; * * p<.01 ; * * * p<.001$

Un segundo análisis comparó los resultados obtenidos en los instrumentos diferenciando entre aquellos casos identificados como clínicos, por haber sido derivados a tratamiento terapéutico $(n=35)$, y el resto de la muestra $(n=103)$, ya que la literatura científica había presentado importantes diferencias según esta variable.

Las diferencias de medias en el grupo de adolescentes que no habían sido derivados a tratamiento muestran puntuaciones más altas en el cuestionario de autoinforme (YSR) frente a la información proporcionada por sus educadores en el CBCL. Estas diferencias entre ambos cuestionarios fueron estadísticamente significativas en todas las escalas y particularmente elevadas para las escalas internalizantes (tabla 3). En cambio, los resultados en el grupo de los adolescentes identificados como clínicos no presentan diferencias significativas en ninguna de las escalas. Es cierto que se aprecia una ligera tendencia hacia puntuaciones más bajas en el YSR en el caso de las escalas de tipo más externalizante (comportamiento disruptivo, conducta agresiva y externalizante), siguiendo la tendencia hallada en otras investigacio- nes. Sin embargo, en la escala de pensamiento y en las de tipo internalizante (ansiedad, depresión, quejas somáticas, e internalizante) el YSR presenta una tendencia hacia puntuaciones mayores.

Los resultados de las correlaciones para el grupo de los adolescentes no clínicos son relativamente elevados para los problemas externalizantes $(r=.57$ en conducta disruptiva, $r$ $=.31$ en conducta agresiva y $r=.45$ en la escala externalizante). El resto de escalas muestra niveles de correlación mucho más bajos.

En el caso del grupo de adolescentes derivados a tratamiento (grupo clínico), las correlaciones entre los dos instrumentos de screening fueron en general más bajas, alcanzando el nivel de significación estadística solamente la escala de conducta disruptiva $(r=.41)$. Por tanto, parece haber una mayor concordancia entre los informantes en las escalas externalizantes (más acusada en el grupo no clínico), habiendo una relación lineal entre sus valoraciones especialmente clara al informar sobre la conducta disruptiva. 
Tabla 3. Diferencias entre medias y correlaciones en CBCL e YSR para adolescentes del grupo clínico y no clínico

\begin{tabular}{|c|c|c|c|c|c|c|c|c|}
\hline & \multicolumn{2}{|c|}{ CBCL } & \multicolumn{2}{|c|}{ YSR } & \multicolumn{2}{|c|}{ Diferencia entre medias } & \multicolumn{2}{|c|}{ Correlación } \\
\hline & $M$ & $D T$ & $M$ & $D T$ & $t / q^{1}$ & $p$ & $r / r h o^{2}$ & $p$ \\
\hline Ansiedad/depresión & 52.51 & 4.87 & 58.59 & 8.43 & -7.02 & $.000^{* * *}$ & .22 & $.029^{*}$ \\
\hline Aislamiento/depresión & 57.07 & 8.47 & 61.41 & 10.3 & -3.70 & $.000^{* * *}$ & .20 & $.041^{*}$ \\
\hline Quejas somáticas & 52.92 & 5.68 & 57.41 & 7.83 & -5.11 & $.000^{* * *}$ & .16 & .102 \\
\hline Probl. sociales & 56.19 & 7.90 & 60.43 & 9.33 & -3.74 & $.000^{* * *}$ & .12 & .236 \\
\hline$\approx$ Probl. de pensamiento & 51.80 & 4.35 & 56.98 & 8.25 & -5.66 & $.000^{* * *}$ & .01 & .909 \\
\hline 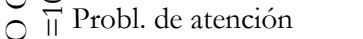 & 56.28 & 6.72 & 59.47 & 8.93 & -3.11 & $.002^{* *}$ & .14 & .161 \\
\hline Z $\Xi$ Conducta disruptiva & 59.53 & 7.97 & 61.83 & 9.05 & -2.93 & $.004^{* *}$ & .57 & $.000^{* * *}$ \\
\hline$\tilde{0}$ Conducta agresiva & 57.15 & 8.75 & 59.64 & 9.38 & -2.37 & $.020^{*}$ & .31 & $.001^{* *}$ \\
\hline Escala internalizante & 49.15 & 10.38 & 58.52 & 10.26 & -6.95 & $.000^{* * *}$ & .12 & .223 \\
\hline Escala externalizante & 55.92 & 10.90 & 60.27 & 10.40 & -3.94 & $.000^{* * *}$ & .45 & $.000^{* * *}$ \\
\hline Escala total & 52.41 & 11.25 & 59.91 & 9.61 & -5.56 & $.000^{* * *}$ & .14 & .143 \\
\hline Ansiedad/depresión & 58.49 & 8.46 & 61.63 & 8.02 & -1.77 & .077 & .16 & .345 \\
\hline Aislamiento/depresión & 60.20 & 9.70 & 60.43 & 8.24 & -0.56 & .574 & .30 & .080 \\
\hline Quejas somáticas & 57.51 & 8.87 & 59.66 & 9.11 & -0.75 & .453 & .08 & .666 \\
\hline$\smile \quad$ Probl. sociales & 63.11 & 8.65 & 62.60 & 8.82 & -0.37 & .707 & .21 & .229 \\
\hline Z $\cong$ Probl. de pensamiento & 57.94 & 8.05 & 59.51 & 7.66 & -0.63 & .531 & .10 & .544 \\
\hline 8 ॥ Probl. de atención & 62.23 & 8.52 & 61.60 & 8.15 & -0.58 & .561 & .14 & .412 \\
\hline$\infty$ Conducta disruptiva & 65.46 & 9.41 & 64.03 & 10.14 & -1.16 & .248 & .41 & $.015^{*}$ \\
\hline Conducta agresiva & 65.94 & 12.65 & 62.71 & 9.48 & -0.84 & .403 & -.05 & .780 \\
\hline Escala internalizante & 57.94 & 10.25 & 61.03 & 10.32 & -1.25 & .212 & .21 & .216 \\
\hline Escala externalizante & 65.43 & 10.48 & 63.11 & 11.34 & -0.91 & .363 & .13 & .457 \\
\hline Escala total & 63.00 & 8.83 & 62.89 & 10.89 & -0.45 & .964 & .10 & .582 \\
\hline
\end{tabular}

${ }^{1}$ Para la muestra no clínica se emplea la prueba " $t$ de Student" y para la clínica la " $z$ de Wilkoxon".

2 Para la muestra no clínica se emplea la " $r$ de Pearson" y para la clínica la "rho de Spearman".

$*: p<.05 ; * * p<.01 ; * * * p<.001$

Tabla 4. Diferencias de medias en CBCL e YSR para adolescentes de los grupos clínico y no clínico.

\begin{tabular}{|c|c|c|c|c|c|c|c|}
\hline & & \multicolumn{2}{|c|}{ CLÍNICO } & \multicolumn{2}{|c|}{ NO CLÍNICO } & \multicolumn{2}{|c|}{ Diferencia entre medias } \\
\hline & & $M$ & $S D$ & $M$ & $S D$ & $t$ & $p$ \\
\hline \multirow{11}{*}{ ن } & Ansiedad/depresión & 58.49 & 8.46 & 52.51 & 4.87 & 3.96 & $.000^{* * *}$ \\
\hline & Aislamiento/depresión & 60.20 & 9.70 & 57.07 & 8.47 & 1.82 & .071 \\
\hline & Quejas somáticas & 57.51 & 8.87 & 52.92 & 5.68 & 2.87 & $.006^{* * *}$ \\
\hline & Probl. sociales & 63.11 & 8.65 & 56.19 & 7.90 & 4.37 & $.000^{* * *}$ \\
\hline & Probl. de pensamiento & 57.94 & 8.05 & 51.80 & 4.35 & 4.30 & $.000^{* * *}$ \\
\hline & Probl. de atención & 62.23 & 8.52 & 56.28 & 6.72 & 4.22 & $.000^{* * * *}$ \\
\hline & Conducta disruptiva & 65.46 & 9.41 & 59.53 & 7.97 & 3.62 & $.000^{* * *}$ \\
\hline & Conducta agresiva & 65.94 & 12.65 & 57.15 & 8.75 & 3.81 & $.000^{* * *}$ \\
\hline & Escala internalizante & 57.94 & 10.25 & 49.15 & 10.38 & 4.34 & $.000^{* * *}$ \\
\hline & Escala externalizante & 65.43 & 10.48 & 55.92 & 10.90 & 4.50 & $.000^{* * *}$ \\
\hline & Escala total & 63.00 & 8.83 & 52.41 & 11.25 & 5.06 & $.000^{* * *}$ \\
\hline \multirow{11}{*}{$\underset{\sim}{\mathscr{N}}$} & Ansiedad/depresión & 61.63 & 8.02 & 58.59 & 8.43 & 1.87 & .064 \\
\hline & Aislamiento/depresión & 60.43 & 8.24 & 61.41 & 10.3 & -0.51 & .608 \\
\hline & Quejas somáticas & 59.66 & 9.11 & 57.41 & 7.83 & 1.41 & .161 \\
\hline & Probl. sociales & 62.60 & 8.82 & 60.43 & 9.33 & 1.21 & .230 \\
\hline & Probl. de pensamiento & 59.51 & 7.66 & 56.98 & 8.25 & 1.60 & .113 \\
\hline & Probl. de atención & 61.60 & 8.15 & 59.47 & 8.93 & 1.25 & .214 \\
\hline & Conducta disruptiva & 64.03 & 10.14 & 61.83 & 9.05 & 1.21 & .230 \\
\hline & Conducta agresiva & 62.71 & 9.48 & 59.64 & 9.38 & 1.67 & .097 \\
\hline & Escala internalizante & 61.03 & 10.32 & 58.52 & 10.26 & 1.25 & .215 \\
\hline & Escala externalizante & 63.11 & 11.34 & 60.27 & 10.40 & 1.37 & .174 \\
\hline & Escala total & 62.89 & 10.89 & 59.91 & 9.61 & 1.53 & .129 \\
\hline
\end{tabular}

Finalmente, la tabla 4 presenta el análisis de diferencias entre el grupo clínico y no clínico para cada instrumento. Para el CBCL los resultados mostraron que el grupo clínico obtuvo puntuaciones medias superiores en todas las escalas, siendo esta diferencia significativa en todas ellas, excepto en la escala de depresión. En el YSR, aunque se aprecia una 
tendencia en esta misma línea (puntuaciones superiores en el grupo clínico frente al grupo no clínico) las diferencias son más pequeñas y en ningún caso resultaron significativas. Así pues los educadores diferencian en sus observaciones claramente al grupo clínico del que no recibe tratamiento, pero los adolescentes no se diferencian del mismo modo en sus respuestas.

Para facilitar una perspectiva global, se presenta la Figura 1 con las medias de todas las escalas, para los dos instrumentos y para los dos grupos (clínico y no clínico). Se puede apreciar que las puntuaciones más altas pertenecen a los ca- sos clínicos en ambos instrumentos, sin que se hayan encontrado diferencias significativas entre ellos en ninguna escala (Tabla 3), contrastando de forma muy visible con los no clínicos del CBCL, que presentan las puntuaciones más bajas en todas las dimensiones. En cambio, los autoinformes (YSR) de los adolescentes sin problemas clínicos se asemejan mucho a los resultados de sus compañeros del grupo clínico (de hecho, ninguna escala presenta diferencias significativas como ya se ha visto en la Tabla 4) y se diferencian significativamente del CBCL no clínico en todas las escalas (Tabla 3).

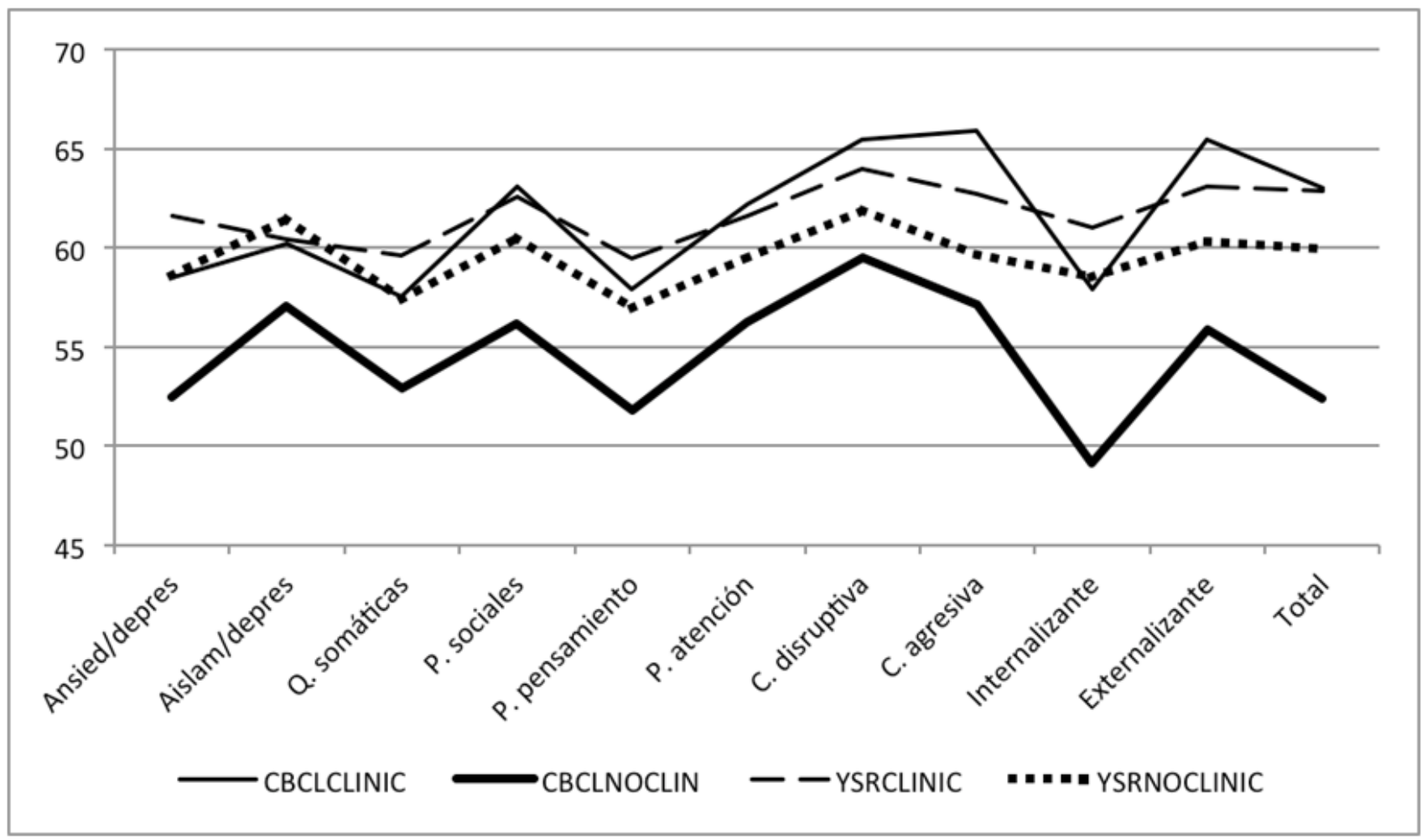

Figura 1. Resultados del CBCL e YSR en ambos grupos clínico y no clínico (puntuaciones T)

\section{Discusión y conclusiones}

La muestra de adolescentes en acogimiento residencial que hemos estudiado presenta una elevada frecuencia (una cuarta parte) de casos derivados a servicios terapéuticos de salud mental. Nuestros datos, aún siendo inferiores a los hallados en otros estudios internacionales de prevalencia de problemas de salud mental en niños y jóvenes de protección (Heflinger, et al., 2000; Keil y Price, 2006; Shin, 2005), indican la necesidad de prestar mucha atención a la detección, evaluación y tratamiento de problemas de salud mental en esta población específica. También es destacable la elevada presencia de casos con discapacidad intelectual (16.7\%), estando casi un tercio de ellos en tratamiento por trastornos emocionales o conductuales, siendo un grupo que reclama mayor investigación sobre sus características y su evolución en acogimiento residencial (Sainero, Del Valle, López, y Bravo, 2013; Tarren-Sweeney y Hazell, 2006).

El grado de concordancia entre la información proporcionada por los educadores en el CBCL y los propios adolescentes en el YSR mostró niveles y patrones de correlación en la línea de lo constatado en investigaciones previas con díadas padres-hijos. Los estudios señalan correlaciones moderadas, con una media de $r=.24$ entre padres e hijos en la mayor revisión efectuada hasta el momento (Achenbach, et al., 1987) y en nuestro caso el promedio de correlaciones de las escalas es de $r=.22$, que es muy similar. No obstante, las diferencias entre escalas son muy notables (oscilando entre .10 y .52) con valores muy superiores en las de tipo externalizante frente a las internalizantes. Parece que los educadores tienen mayores dificultades para apreciar los problemas internalizantes que los adolescentes a su cuidado manifiestan en el autoinforme $y$, en cambio, obtienen una razonable 
concordancia con los problemas externalizantes. Esta diferencia se suele encontrar también en los padres, ya que los trastornos externalizantes son más fácilmente objetivables y medibles desde la perspectiva del adulto que los internalizantes, como han señalado diversos estudios (Achenbach et al., 1987; Cantwell, Lewinsohn, Rohde, y Seeley, 1997; Edelbrock, Costello, Dulcan, Conover, y Kala, 1986) que inciden en la importancia que tiene la saliencia de la conducta en la detección de comportamientos clínicos (Karver, 2006).

Aunque el grado de concordancia así medido tiene un indudable interés, la literatura científica ha señalado que las diferencias entre informantes dependen claramente de que se trate de adolescentes derivados a servicios clínicos o no. Nuestros resultados permiten confirmar los hallazgos de investigaciones internacionales acerca de que existirían puntuaciones más altas en problemas informados por los jóvenes no identificados como clínicos frente a la proporcionada por sus padres (en nuestro caso educadores), con diferencias significativas en todas las escalas del instrumento, tanto específicas como las de segundo orden. Este hecho, ya constatado por la mayoría de los estudios con muestras de población general y no clínica (Rescorla et al., 2007) parece responder a las dificultades de identificación de algunas conductas, con especial incidencia en las más privadas, y/o al cambio en el comportamiento en función del contexto (Youngstrom, Loeber, y Stouthamer-Loeber, 2000), aunque lo cierto es que la investigación carece de explicaciones definitivas acerca de esta discrepancia (De los Reyes y Kazdin, 2005). Lo destacable en nuestro caso es que los instrumentos funcionan con educadores de forma similar a los padres, lo que de algún modo permite justificar su uso.

En cambio, los resultados para el grupo clínico son discrepantes con la literatura científica que señalaba que, en este caso, son los adultos los que informan de mayor problemática. Los jóvenes de nuestra muestra que se hallaban en tratamiento no presentaron diferencias significativas con sus educadores. La Figura 1 permite visualizar una tendencia a que en los problemas internalizantes los jóvenes siguen puntuando por encima de los adultos, mientras en los de tipo externalizante sí se observa que los educadores reportan mayores indicadores de trastorno. En cualquier caso, como hemos indicado, no alcanzan la significación estadística en ninguna escala.

La justificación que diversos autores ofrecen para que los padres informen de más problemas en el caso de muestra clínica es que la monitorización de la conducta de los adolescentes, una vez que han sido identificados como "clínicos o con conducta problemática", lleva a los informantes adultos (padres) a un análisis más fino de su comportamiento y por tanto a una mayor tasa de problemas informados (Huberty, Austin, Harezlak, Dunn, y Ambrosius, 2000). Frente a esto, otros autores señalan que los adolescentes de este grupo tienen un sesgo o dificultad en la identificación de su sintomatología una vez que están a tratamiento (Salbach-Andrae, Klinkowski, Lenz, y Lehmkuhl, 2009). En nuestro estudio podría ocurrir que los adolescentes tengan mayor conciencia de sus problemas, ya que se encuentran en un hogar de acogida, en una situación de por sí ya diferenciada del resto de jóvenes y bajo una intervención social protectora. Por otra parte, también cabría la hipótesis de que los educadores, a diferencia de los padres, estén mucho más habituados a observar comportamientos problemáticos de un gran número de adolescentes a su cuidado y sean más ponderados en sus puntuaciones sobre el grupo clínico. Los problemas de observación y detección de trastornos de conducta en acogimiento residencial pueden tener un impacto muy negativo en el desarrollo de los niños y jóvenes atendidos. Al tratarse de casos que han pasado por experiencias muy traumáticas relacionadas con diferentes formas de maltrato infantil en sus familias, la probabilidad de presentar problemas emocionales, conductuales y de desarrollo es muy elevada (Moreno, García- Baamonde y Blázquez, 2010).

De acuerdo con los resultados obtenidos parece que las manifestaciones problemáticas son informadas de manera más clara por los educadores en aquellos casos que ya están siendo tratados por servicios de atención clínica (tratamiento psicológico y/o psiquiátrico) frente a quienes no presentan esta intervención. Este hecho, podría explicarse por ser habitualmente los educadores los que solicitan las derivaciones a tratamiento de aquellos niños en los que observan problemas emocionales o conductuales. De algún modo, ratifican mediante la técnica de screening lo que fue en su momento una observación más informal y que determinó la derivación a tratamiento. Es muy importante tener en cuenta que la falta de uso de este tipo de instrumentos puede estar limitando la derivación a tratamiento a casos más fácilmente detectables y particularmente a los trastornos externalizantes en detrimento de los problemas internalizantes.

Efectivamente, los datos sobre la incidencia de problemáticas en esta muestra señalan la dominancia de los trastornos de tipo externalizante frente a los internalizantes, algo acorde a lo constatado en los diferentes estudios realizados con niños y adolescentes del sistema de protección (Garland et al., 2003; Heflinger et al., 2000; Keil y Price, 2006; Martin, García y Siveiro, 2012). Sin embargo, los resultados indican claramente que parece existir un notable malestar emocional y conductual informado por el grupo de jóvenes no clínicos que no alcanza la misma gravedad desde la perspectiva de los educadores. Esto es especialmente visible en los trastornos internalizantes, donde los jóvenes alcanzan puntuaciones mucho más altas que las de sus educadores. Dados los citados problemas de detección y derivación a servicios terapéuticos es importante que los educadores tengan la formación y las técnicas para evaluar y detectar tempranamente las necesidades de salud mental que presentan estos jóvenes, con especial incidencia en las conductas más privadas y menos objetivables. Por otra parte, el uso del YSR, tal y como ha quedado de manifiesto, debe ser considerado un complemento muy importante al aportar la perspectiva de los propios jóvenes de modo que los criterios no sean exclusivamente las observaciones de los adultos. Sólo tomando en consideración la complementariedad de los dos instrumen- 
tos, obtendremos la información más completa posible y minimizaremos las probabilidades de sesgo por parte de algunos informantes. Esta identificación inicial de manifestaciones clínicas, deberá completarse posteriormente con instrumentos específicos de evaluación diagnóstica.

La implantación de instrumentos de screening como los utilizados en esta investigación, en el marco de los hogares de protección, puede facilitar una detección de la sintomatología existente, además de permitir monitorizar la evolución conductual y emocional del joven durante su tiempo en protección (véase una propuesta en Del Valle, Sainero, y Bravo (2011). Con ello se podría evitar un posible sesgo hacia una sobrevaloración de problemas externalizantes en detrimento de problemas internalizantes que pasan más desapercibidos y podrían quedar sin tratar terapéuticamente.

Dadas las condiciones de especial vulnerabilidad de estos jóvenes, estudios como el actual deberían reforzar la necesidad de establecer sistemas de detección y monitorización que aseguren la agilidad y la eficacia de la intervención protectora y terapéutica, contando con la perspectiva de educadores y jóvenes acogidos durante el tiempo en que éstos se encuentran en protección, de forma previa a su egreso y a las

\section{Referencias}

Achenbach, T. M. (2006). As Others See Us : Clinical and Research Implications of Cross-Informant Correlations for Psychopathology. Current Directions in Psychological Science, 15(2), 94-98.

Achenbach, T. M., McConaughy, S. H., y Howell, C. T. (1987). Child/Adolescent behavioral and emotional problems: Implications of cross-informant correlations for situational specificity. Psychological Bulletin, 101(2), 213-232.

Achenbach, T. M., y Rescorla, L. (2001). Manual for the ASEBA school-age forms y profiles. Burlington, VT: University of Vermmont, Research Center for Children Youths y Families.

Achenbach, T. M., y Edelbrock, C. (1983). Manual for the Child Behavior Check List and Revised Child Behavior Profiles. Burlington, VT: University of Vermont, Department of Psychiatry.

Barkmann, C., y Schult-Markwort, M. (2005). Emotional and behavioral problems of children and adolescents in Germany: An epidemiological screening. Social Psychiatry and Psychiatric Epidemiology, 40(5), 357-366.

Begovac, I., Rudan, V., Skocic, M., y Filipovic, O. (2004). Comparisons of self-reported and parent-reported emotional and behavioral problems in adolescents from Croatia. Collegium Antropologicum, 28(1), 393-401.

Berg-Nielsen, T. S., Vika, A., y Dahl, A. A. (2003). When adolescents disagree with their mothers: CBCL-YSR discrepancies related to maternal depression and adolescent self-esteem. Child: Care, Health and Development, 29(3), 207-213.

Bravo, A., y Del Valle, J. F. (2009). Crisis y revisión del acogimiento residencial: su papel en la protección infantil. Papeles del Psicólogo, 30(1), 42-52. (2009).

Bronsard, G., Lançon, C., Loundou, A., Auquier, P., Rufo, M., y Siméoni, M. (2011). Prevalence rate of DSM mental disorders among adolescents living in residential group homes of the French Child Welfare System Children and Youth Services Review, 33(10), 1886-1890.

Burns, B. J., Phillips, S., Wagner, H. R., Barth, R., Kolko, D., Campbell, Y., et al. (2004). Mental health need and access to mental health services by youths involved with child welfare: A national survey. Journal of the American Academy of Childy Adolescent Psychiatry, 43(8), 960-970.

Cantwell, D. P., Lewinsohn, P. M., Rohde, P., y Seeley, J. R. (1997). Correspondence between adolescent report and parent report of psychiatric diagnostic data. Journal of the American Academy of Child and Adolescent Psychiatry, 36(5), 610-619. complicaciones añadidas asociadas a esta transición (López, Santos, Bravo, y Del Valle, 2013).

Como limitaciones de nuestra investigación es importante señalar que la muestra objeto de este estudio procede de una única comunidad autónoma española por lo que se debe tener mucha cautela a la hora de generalizar los resultados. Además, el tamaño muestral del grupo clínico es relativamente pequeño (a pesar de suponer un porcentaje muy elevado con respecto al total de jóvenes en acogimiento residencial).

Por otra parte, el haber dispuesto de una muestra compuesta por todos los adolescentes en acogimiento residencial de una comunidad autónoma, incluyendo hogares públicos y privados, de pequeño y de gran tamaño, especializados en adolescentes y dedicados a edades diferentes, etc., evita sesgos que con frecuencia se cometen cuando se muestrea un número de hogares determinado y sus características no cubren todo el espectro posible. Creemos que esta es una posible explicación para la diversidad de resultados que nacional e internacionalmente se encuentran en ocasiones cuando se investiga en acogimiento residencial.

Castro-Fornieles, J. (2013). Psiquiatría del Niño y del Adolescente: necesidad de formación y desarrollo. Revista de Psiquiatría y Salud Mental, 6(2), 5759. Doi: http://dx.doi.org/10.1016/i.rpsm.2012.10.001

Clausen, J. M., Landsverk, J., Ganger, W., Chadwick, D., y Litrownik, A. (1998). Mental health problems of children in foster care. . Journal of Child and Family Studies, 7(3), 283-296.

De los Reyes, A., y Kazdin, A. E. (2005). Informant discrepancies in the assessment of childhood psychopathology: A critical review, theoretical framework, and recommendations for further study. Psychological Bulletin, 131(4), 483-509.

Defensor del Pueblo. (2009). Centros de protección menores con trastornos de conducta y en situación de dificultad social [Residential child care for children with behavioral diseases and social difficulties]. Madrid, Spain: Defensor del Pueblo.

Del Valle, J. F., Bravo, A., y López, M. (2009). El acogimiento familiar en España: implantación y retos actuales. Papeles del Psicólogo, 30(1), 33-41.

Del Valle, J. F., Sainero, A., y Bravo, A. (2011). Salud mental de menores en acogimiento residencial. Badajoz, España: Junta de Extremadura.

Edelbrock, C., Costello, A. J., Dulcan, M. K., Conover, N. C., y Kala, R. (1986). Parent-child agreement on child psychiatric symptoms assessed via structured interview. Journal of Child Psychology and Psychiatry, 27(2), 181-190.

European Association for Research into Residential Child Care. (1998). Care to listen: A report of residential child care in four European countries. In The Center for Residential Child Care. Glasgow..

Farmer, E. M. Z., Burns, B. J., Chapman, M. V., Phillips, S., Angold, A., y Costello, E. (2001). Use of mental health services by youth in contact with social services. Social Services Review, 75(4), 605-624.

Ferdinand, R. F., Van der Ende, J., y Verhulst, F. C. (2004). Parentadolescent disagreement regarding psychopathology in adolescent from the general population as a risk factor for adverse outcome. Journal of Abnormal Child Psychology, 113(2), 198-206.

Ford, T., Vostanis, P., Meltzer, H., y Goodman, R. (2007). Psychiatric disorder among British children looked after by local authorities: A comparison with children living in private households. British Journal of Psychiatry, 190, 319-325. 
Garland, A. F., Landsverk, J. A., y Lau, A. S. (2003). Racial and ethnic disparities in mental health service use among children in foster care. Children and Youth Services Review, 25(5-6), 491-507.

Handwerk, M. L., Larzelere, R. E., Soper, S. H., y Friman, P. C. (1999). Parent and Child Discrepancies in Reporting Severity of Problem Behaviors in Three Out-of-Home Settings. Psychological Assessment, 11(1), 14-23.

Heflinger, C. A., Simpkins, C. G., y Combs-Orme, T. (2000). Using the CBCL to determine the clinical status of children in state custody. Children and Youth Services Review, 22(1), 55-73.

Holtan, A., Ronning, J., Handegard, B., y Sourander, A. (2005). A comparison of mental health problems in kinship and non kinship foster care. European Child y Adolescent Psychiatry, 14(4), 200-207.

Huberty, T. J., Austin, J. K., Harezlak, J., Dunn, D. W., y Ambrosius, W. T. (2000). Informant Agreement in Behavior Ratings for Children with Epilepsy. Epilepsi and Behavior, 1(6), 427-435.

Hukkanen, R., Sourander, A., Santalahti, P., y Bergroth, L. (2005). Have psychiatric problems of children in children's homes increased? Nordic Journal of Psychiatry, 59(6), 481-485.

Karver, M. S. (2006). Determinants of Multiple Informant Agreement on Child and Adolescent Behavior. Journal of Abnormal Child Psychology, 34(2), 242-253. Doi: 10.1007/s10802-005-9015-6

Keil, V., y Price, J. M. (2006). Externalizing behavior disorders in child welfare settings: Definition,prevalence, and implications for assessment and treatment. Children and Youth Services Review, 28(7), 761-779.

Kolko, D., y Kazdin, A. E. (1993). Emotional/behavioral problems in clinic and nonclinic children: Correspondence among child, parent and teacher reports. Journal of Child Psychology and Psychiatry, 34(6), 991-1006.

Kristensen, H. (2001). Multiple Informants' report of emotional and behavioral problems in a nation-wide sample of selective mute childrens and control. European Child and Adolescent Psychiatry, 10(2), 135-142.

Landsverk, J., Burns, B. J., Stambaugh, L., y Rolls-Reutz, J. (2006). Mental health care for children and adolescents in foster care. Review of research literature. Seattle, WA: Casey Family Programs.

López, M., Santos, I., Bravo, A., \& Del Valle, J. F. (2013). El proceso de transición a la vida adulta de jóvenes acogidos en el sistema de protección. Anales de Psicología, 29(1), 187-196.

Martín, E., García, M., y Siverio, M. (2012). Inadaptación autopercibida de los menores en acogimiento residencial. Anales De Psicología, 28(2), $541-$ 547. Doi:10.6018/analesps.28.2.148921

Moreno, J. M., García-Baamonde, M. E., y Blázquez, M. (2010). Desarrollo lingüístico y adaptación escolar en niños en acogimiento residencial. Anales de Psicología, 26(1), 189-196.

Muela, A., Balluerka, N., y Torres, B. (2013). Ajuste social y escolar de jóvenes víctimas de maltrato infantil en situación de acogimiento residencial. Anales De Psicologia, 29(1), 197-206. Doi:10.6018/analesps.29.1.124941

Nicholas, B., Roberts, S., y Wurr, S. (2003). Looked after children in residential homes. Child and Adolescent Mental Health, 8(2), 78-83.

Observatorio de la Infancia. (2012). Estadística básica de medidas de protección a la infancia [Basic statistics of child care interventions] (Vol. 14). Madrid, Spain: Ministerio de Sanidad, Políticas Sociales e Igualdad

Pecora, P. J., Jensen, P. S., Romanelli, L. H., Jackson, L., y Ortiz, A. (2009). Mental health services for children placed in foster care: An overview of current challenges. Child Welfare, 88(1), 1-25.

Renk, K., y Phares, V. (2004). Cross-informant ratings of social competence in children and adolescents. Clinical Psychology Review, 24(2), 239-254.

Rescorla, L., Achenbach, T. M., Ivanova, M. Y., Dumenci, L., Almqvist, F., Bilenberg, N., et al. (2007). Behavioral and emotional problems reported by parents of children ages 6 to 16 in 31 societies. Journal of Emotional y Behavioral Disorders, 15(3), 130-142.
Rescorla, L., Ginzburg S, Achenbach TM, Ivanova MY, Almqvist F, Begovac I, et al. (2013). Cross-informant agreement between parentreported and adolescent self-reported problems in 25 societies. Journal of Clinical Child y Adolescent Psychology, 42(2), 262-273.

Rey, J. M., Schrader, E., y Morris-Yates, A. (1992). Parent-child agreement on children's behavior reported by the Child Behavior Checklist (CBCL). Journal of Adolescence, 15(3), 219-230.

Sainero, A., Bravo, A., \& Del Valle, J. F. (2014). Examining needs and referrals to mental health services for children in residential care in Spain: an empirical study in an autonomous community. Journal of Emotional and Behavioral Disorders, 22(1), 16-26.

Sainero, A., Del Valle, J. F., López, M., y Bravo, A. (2013). Exploring the specific needs of an understudied group: children with intellectual disability in residential child care. Children and Youth Services Review, 35(9), 1393-1399.

Salbach-Andrae, H., Klinkowski, N., Lenz, K., y Lehmkuhl, U. (2009). Agreement between youth-reported and parent-reported psychopathology in a referred sample. European Child y Adolescent Psychiatry, 18(3), 136-143. Doi: 10.1007/s00787-008-0710-z

Seiffge-Krenke, I., y Kollmar, F. (1998). Discrepancies between Mothers' amd Fathers' Perceptions of Sons' and Daughters' Problem Behavior: A Longitudinal Analysis of Parent- Adolescents Agreement on Internalising and Externalising Problem Behavior. Journal of Child Psychology and Psychiatry, 39(5), 687-697.

Sempik, J., Ward, H., y Darker, I. (2008). Emotional and behavioural difficulties of children and young people at entry into care. Clinical Child Psychology and Psychiatry, 13(2), 221-233.

Shin, H. S. (2005). Need for and actual use of mental health service by adolescents in the child welfare system. Children and Youth Services Review, 27(10), 1071-1083.

Sinclair, I., Baker, C., Lee, J., y Gibbs, I. (2007). The Pursuit of Permanence: a Study of the English Care System.Quality Matters in Children's Services. Series London: Jessica Kingsley

Stranger, C., y Lewis, M. (1993). Agreement among parents, teachers, and children on internalizing and externalizing behavior problems. Journal of Clinical Child Psychology, 22(1), 107-115.

Tarren-Sweeney, M., y Hazell, P. (2006). Mental health of children in foster and kinship care in New South Wales, Australia. Journal of Paediatrics and Child Health, 42(3), 89-97.

Van der Ende, J., y Verhulst, F. C. (2005). Informant, gender and age differences in ratings of adolescent problem behavior. European Childy Adolescent Psychiatry, 14(3), 117-126.

Verhulst, F. C., y Van der Ende, J. (1992). Agreement between parent's report and adolescents' self reports of problem behavior. Journal of Child Psychology and Psychiatry, 33(6), 1011-1023.

Vierhaus, M., y Lohaus, A. (2008). Children and Parents as Informants of Emotional and Behavioural Problems Predicting Female and Male Adolescent Risk Behaviour: A Longitudinal Cross-Informant Study. Journal of Youth y Adolescence, 37(2), 211-224. Doi: 10.1007/s10964-0079193-3

Yeh, M., y Weisz, J. R. (2001). Why are we here at the clinic? Parent-child (dis)agreement on referral problems at outpatient treatment entry. Journal of Consulting and Clinical Psychology, 69(6), 1018-1025.

Youngstrom, E., Loeber, R., y Stouthamer-Loeber, M. (2000). Patterns and correlates of agreement between parent, teacher, and male adolescent ratings of externalizing and internalizing problems. Journal of Consulting and Clinical Psychology, 68(6), 1038-1050.

(Articulo recibido: 06-09-2013; revisado: 14-01-2014; aceptado: 17-02-2014) 